\title{
STRUCTURES AND REACTIVITIES OF ORGANOLITHIUM COMPOUNDS
}

\author{
THEODORE L. BROWN \\ Noyes Chemical Laboratory, University of Illinois, \\ Urbana, Illinois 61801, U.S.A.
}

\begin{abstract}
The characteristic species present in organolithium compounds under various conditions are reviewed. The effects of environment on the kinetics and energetics of exchange reactions are discussed. A summary is given of various pathways by which organolithium compounds might react. Kinetic and spectroscopic evidence for these processes under various conditions are reviewed. A discussion is presented of the significance of certain results obtained by chemically induced dynamic nuclear polarization (CIDNP).
\end{abstract}

THE extensive researches on organolithium compounds of the past decade provide a basis for understanding in detail the chemistry of these compounds. The purpose of this contribution is to review the various lines of evidence regarding the constitution of organolithium reagents, and to relate these to various possible pathways by which they might undergo chemical reaction.

The x-ray structural work of Dietrich ${ }^{1}$ (ethyllithium), Weiss and Lucken ${ }^{2}$ (methyllithium) and Stucky and Patterman ${ }^{3}$ (benzyllithium) provide a basis for evaluation of models for the structures of the organolithium compounds in solutions. The order of volatilities, $\mathrm{CH}_{3} \mathrm{Li} \ll \mathrm{C}_{2} \mathrm{H}_{5} \mathrm{Li}<\left(\mathrm{CH}_{3}\right)_{2} \mathrm{CHLi}<$ $\left(\mathrm{CH}_{3}\right)_{3} \mathrm{CLi}$, suggests that there is significant association between the structural units in the solid, provided that steric interactions do not interfere. From this point of view, the structure of tert-butyllithium would provide the ideal example of bonding within the characteristic oligomeric unit, in this case, a tetramer. Unfortunately, the solid appears to have an enormous unit cell ${ }^{4}$. In addition, it is extremely reactive with oxygen, and alkoxide purity is a serious problem.

In solution the characteristic structural units observed are the hexamer, tetramer, dimer and monomer, depending on the nature of the organic group and the nature of the solvent ${ }^{5}$. The prevailing structures in hydrocarbon solvents are hexamer and tetramer when the organic group is saturated aliphatic. Hexameric association is found when the alkyl group is primary; tert-butyllithium, on the other hand, is tetrameric. For intermediate degrees of steric interactions between alkyl groups it may happen that the free energy difference between hexamer and tetramer is small, so that an equilibrium between the two forms is seen, as with trimethylsilylmethyllithium, in benzene ${ }^{6}$. The associations of organolithium compounds in basic solvents such as ethers, 
THEODORE L. BROWN

amines, etc., are difficult to ascertain, because of reactivity of the organolithium reagent toward the solvent. West and Waack have recently reported ${ }^{7}$ valuable osmometry studies of the associations of some organolithium compounds in ether and THF. These results, along with some others, are summarized in Table 1.

Table 1. Characteristic associations of organolithium compounds

\begin{tabular}{|c|c|c|c|}
\hline Bridging group & Vapour & Hydrocarbon & Basic solvent \\
\hline$-\mathrm{OC}\left(\mathrm{CH}_{3}\right)_{3}$ & $6^{11}$ & 6 & - \\
\hline$-\mathrm{CH}_{3}$ & & - & $4^{7}$ \\
\hline$-\mathrm{C}\left(\mathrm{CH}_{3}\right)_{3}$ & $4^{12}$ & $4^{6,9}$ & - \\
\hline$-\mathrm{CH}_{2} \mathrm{CH}_{2} \mathrm{CH}_{2} \mathrm{CH}_{3}$ & - & $6^{6}$ & $4^{7}$ \\
\hline$-\mathrm{CH}_{2} \mathrm{Si}\left(\mathrm{CH}_{3}\right)_{3}$ & $4^{11}$ & $4-6^{6}$ & - \\
\hline$-\mathrm{C}_{6} \mathbf{H}_{5}$ & - & - & $2^{7}$ \\
\hline$-\mathrm{N}\left[\mathrm{Si}\left(\mathrm{CH}_{3}\right)_{3}\right]_{2}$ & - & $2+^{8}$ & $1+8$ \\
\hline
\end{tabular}

Mass spectrometry has provided valuable supporting evidence for the colligative property measurements in solutions ${ }^{10-12}$. Although hexamers are not a major species for ethyllithium ${ }^{10}$, they are a significant fraction of the vapour. For trimethylsilylmethyllithium ${ }^{11}$ and tert-butyllithium ${ }^{12}$ tetramers are the predominant vapour form.

Nuclear magnetic resonance spectroscopy is a valuable tool for study of organolithium compounds in solution. Study of the low temperature ${ }^{7} \mathrm{Li}$ spectra of mixtures of organolithium compounds provides a means for learning about the degree of association and structure of the associated unit. When two organolithium compounds which exhibit ${ }^{7} \mathrm{Li}$ resonances at different chemical shifts are mixed together in a solvent, exchange reactions may occur, leading to formation of a range of mixed organolithium tetramers ${ }^{13,14}$. This is illustrated, for the mixing of two tetrameric species, in Figure 1. If the ${ }^{7} \mathrm{Li}$ chemical shifts characteristic of the two pure organolithium compounds are sufficiently different, a multiplet in the ${ }^{7} \mathbf{L i}$ spectrum
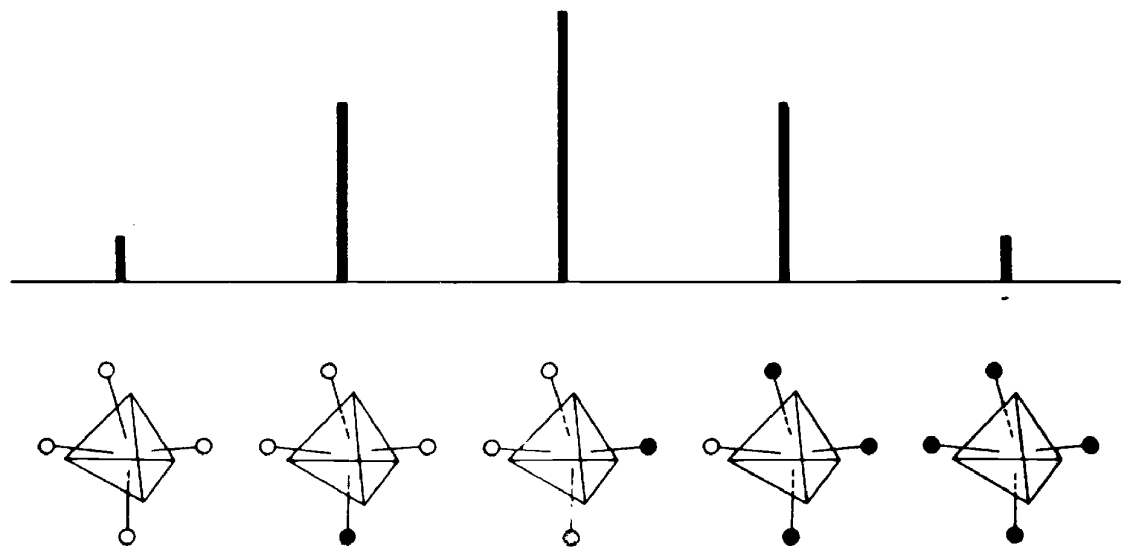

Figure 1. Relative concentrations and structures of the five tetrameric species formed in a $1: 1$ mixture of $(\mathrm{LiR})_{4}$ and $\left(\mathrm{LiR}^{\prime}\right)_{4}$, assuming random distributions of the alkyl groups, which are represented by the black and white circles. (Taken from ref. 14.) 
is seen, characteristic of the lithiums which experience the various environments of neighbouring alkyl groups. On the basis of a local environment assumption $^{13}$, i.e. that the chemical shift of the lithium is an average of the effects created by the three neighbouring alkyl groups, it has been possible to conclude that the simple alkyllithium compounds are tetrameric in ether. The same line of reasoning has been of use in analysing the ${ }^{7} \mathrm{Li}$ and ${ }^{13} \mathrm{C}$ INDOR spectra of ${ }^{13} \mathrm{C}$-enriched methyllithium ${ }^{15}$, and tert-butyllithium ${ }^{16}$. On the basis of the similarities observed in the ${ }^{7} \mathrm{Li}$ spectra of ${ }^{13} \mathrm{C}$-enriched methyllithium in THF and triethylamine at $-80^{\circ} \mathrm{C}^{15}$, it is possible to conclude that methyllithium is tetrameric in both solvents at this temperature.

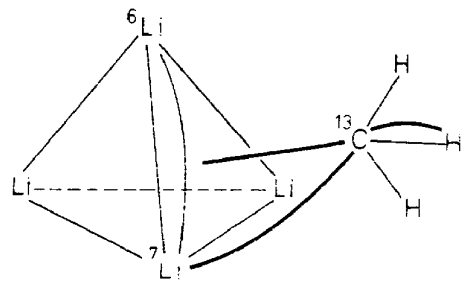

$$
\begin{array}{ll}
J\left({ }^{7} \mathrm{~L} i-{ }_{\mathrm{Li}}\right)<0.3 \mathrm{~Hz} & J\left({ }^{13} \mathrm{C}-{ }^{7} \mathrm{Li}\right)=15 \mathrm{~Hz} \\
J\left({ }^{13} \mathrm{C}-{ }^{1} \mathrm{H}\right)=97 \mathrm{~Hz}
\end{array}
$$

Figure 2. Scalar couplings observed for methyllithium in ether solution.

The magnitudes of the various possible scalar couplings in methyllithium ether solution provide interesting data which relate to the bonding in organolithium moieties (Figure 2). The fact that there is an appreciable ${ }^{7} \mathrm{Li}-{ }^{13} \mathrm{C}$ scaler coupling $\left(15 \mathrm{~Hz}\right.$ in methyllithium ${ }^{15}, 11 \mathrm{~Hz}$ in tert-butyllithium $\left.{ }^{16}\right)$ is indication of an appreciable covalency in the carbon-lithium bond.

On the other hand, the absence of an observable ${ }^{6} \mathrm{Li}-{ }^{7} \mathrm{Li}$ coupling ${ }^{17}$ suggests that there is little valence electron charge density in the region of the $\mathrm{Li}-\mathrm{Li}$ bond axes. The low value observed for the ${ }^{13} \mathrm{C}-{ }^{1} \mathrm{H}$ scaler coupling is consistent with the idea that the lithium-carbon bond is quite polar, and that the carbon carries a significant negative charge. The estimate of McKeever, Waack, Doran and Baker ${ }^{15}$, about $-0 \cdot 1 \mathrm{e}^{-}$, is, I believe, too small. This estimate is based on the chemical shift of the ${ }^{13} \mathrm{C}$ resonance in methyllithium. The ${ }^{13} \mathrm{C}-{ }^{1} \mathrm{H}$ coupling, on the other hand, would suggest a larger negative charge, since the scalar coupling decreases with increasing negative charge on carbon in $\mathrm{CH}_{3} \mathrm{X}$ compounds. In $\left[\left(\mathrm{CH}_{3}\right)_{3} \mathrm{Al}\right]_{2}$ the average coupling constant of bridge and terminal methyl groups is $113 \mathrm{~Hz}$; in $\operatorname{LiAl}\left(\mathrm{CH}_{3}\right)_{4}$, in ether solution, it is $108 \mathrm{~Hz}$. In both these cases, the charge on carbon would be expected to be larger than $0 \cdot 1 \mathrm{e}^{-}$. It must be admitted, however, that the comparison of either chemical shift or coupling constant with carbon in other types of environments is tenuous, and hardly provides a basis for quantitative estimation of charge.

Having once established the type of bonding and the prevalent oligomeric 
species present in a given solvent system, the next questions of interest relate to the lability of these species, i.e. to their proclivities for exchange through dissociative or, possibly, associative, processes. It is convenient to consider first the behaviour of tetrameric species. Exchanges of magnetic environments of the lithium atoms of the tetramers, observed via the ${ }^{7}$ Li n.m.r. spectra, is presumed to occur via dissociation of the tetramer. The process

$$
\mathrm{R}_{4} \mathrm{Li}_{4} \rightarrow 2 \mathrm{R}_{2} \mathrm{Li}_{2}
$$

with recombination of dimers to reform the tetramers, is not readily distinguished from the case in which the dimers further dissociate to monomers more rapidly than they recombine to form tetramer. Indeed, the dissociation of tetramers directly to monomers is a possibility which cannot at present be ruled out. Nevertheless, it seems reasonable to assume that, because dimers are a relatively stable sub-unit of the parent tetramer, the tetramer dissociates as in equation 1. We leave open the question of whether dimers rapidly dissociate to form monomers.

On the basis of n.m.r. studies of organolithium exchange processes, it has been possible to assess the kinetics and energetics of the dissociation of methyllithium in ether ${ }^{18}$. The process

$$
\left(\mathrm{CH}_{3} \mathrm{Li}\right)_{4} \rightarrow 2\left(\mathrm{CH}_{3} \mathrm{Li}\right)_{2}
$$

occurs with an enthalpy of activation, $\Delta H^{\ddagger}$, of $11 \mathrm{kcal} / \mathrm{mole}$ and an entropy of activation, $\Delta S^{\ddagger}$, of $-6 \mathrm{cal} / \mathrm{deg}$-mole. Ideally, these quantities should be compared with those for methyllithium in a variety of other solvents in which it is tetrameric. Unfortunately, the solubility of methyllithium in hydrocarbon solvents is negligible, and it would no doubt be a hexamer, or even more highly associated, in a hydrocarbon solvent. For reasons which are explained later, the only compound which is in fact suitable for evaluation of the tetramer dissociation equilibrium in hydrocarbon solution is tert-butyllithium. The dissociation of tert-butyllithium has been studied ${ }^{12}$ using n.m.r. spectroscopy to follow the approach to equilbrium with another organolithium compound. The kinetic parameters for the process

$$
\left[\left(\mathrm{CH}_{3}\right)_{3} \mathrm{CLi}\right]_{4} \rightarrow 2\left[\left(\mathrm{CH}_{3}\right)_{3} \mathrm{CLi}\right]_{2}
$$

in cyclopentane solution are $\Delta H^{\ddagger}=24 \mathrm{kcal} / \mathrm{mole}, \Delta S^{\ddagger} \sim 0 \mathrm{cal} / \mathrm{deg}-\mathrm{mole}$.

The tert-butyllithium dissociation is very much slower than the analogous process for methyllithium in ether. The presumption that the exchange of $t$-butyllithium with another organolithium compound involves rupture of the tetrameric $\mathrm{Li}_{4}$ core rather than, for example, a process of the form

$$
\mathrm{R}_{4} \mathrm{Li}_{4} \rightarrow \mathrm{R}_{3} \mathrm{Li}_{4}^{+}+\mathrm{R}^{-}
$$

can be tested by a mass spectrometric experiment. Samples of isotopically pure tert-butyllithium- 6 and tert-butyllithium-7 are mixed in cyclopentane, and then solvent removed after varying periods of time. An exchange which occurred without rupture of the $\mathrm{Li}_{4}$ core would not lead to formation of tetramers of intermediate mass numbers. In the mass spectrum of the sample after solvent removal, the mass peaks of the $\mathrm{R}_{3} \mathrm{Li}_{4}^{+}$ions thus reveal the presence of exchange leading to species of the form ${ }^{6} \mathrm{Li}_{n}{ }^{7} \mathrm{Li}_{4-n} \mathrm{R}_{4}, n=1,2,3$. 
$0.3 \mathrm{~h}$
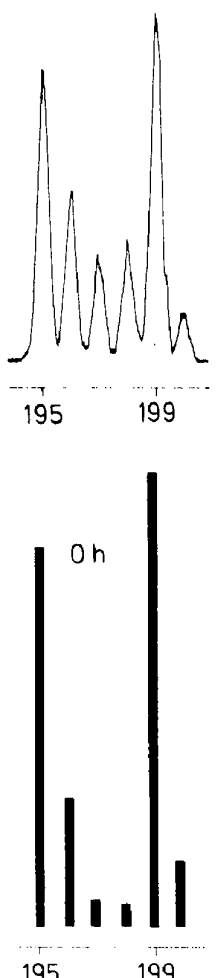

$12 \cdot 2 \mathrm{~h}$

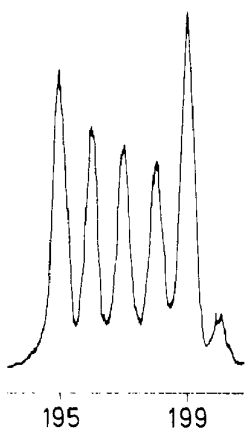

$\infty \mathrm{h}$
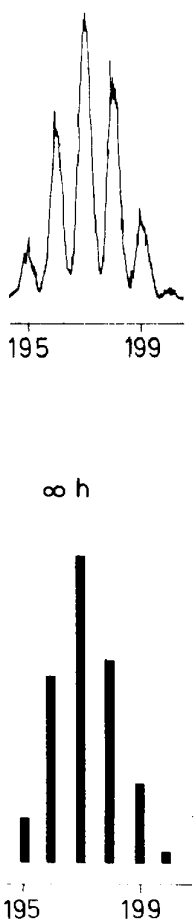

Figure 3. Mass spectra of mixtures of $\left(\mathrm{CH}_{3}\right)_{3} \mathrm{C}^{6} \mathrm{Li}$ and $\left(\mathrm{CH}_{3}\right)_{3} \mathrm{C}^{7} \mathrm{Li}$ taken from cyclopentane solution (ref. 12)

Figure 3 shows a pair of mass spectra, along with calculated spectra for zero and complete exchange. Spectra of samples from which solvent has been removed after varying periods of time exhibit distributions of intermediate mass peaks which are in agreement with expectations based on the n.m.r. results.

The results so far discussed indicate that dissociation of the tert-butyllithium tetramer in cyclopentane requires considerably more energy than dissociation of the methyllithium tetramer in ether. It would be very helpful if the tert-butyllithium tetramer dissociation could be followed in ether or other pure basic solvent, so that the tert-butyllithium and methyllithium systems could be compared under identical conditions. Unfortunately, efforts in this direction have so far been blocked by the extreme reactivity of tertbutyllithium with basic solvents, and the difficulties of designing an appropriate exchange systems which can yield the desired information. Nevertheless, it would seem reasonable to conclude that most of the difference in energetics of the tetramer dissociations is attributable to the effect of solvent. The more electron-releasing character of the tert-butyl group might be expected to lead 
to some stabilization of the tetramer, but on the other hand its greater steric requirement should lead to some acceleration of dissociation.

The effect of added base on the dissociation of the tert-butyllithium tetramer has been noted only qualitatively ${ }^{6}$, but it is clear that the effects are very great. Addition of triethylamine in the proportion of about one base per tert-butyllithium tetramer leads to an acceleration of at least 50 -fold in the dissociation. On the basis of colligative property and ${ }^{7} \mathrm{Li}$ chemical shift studies (Figure 4) it does not appear that a base such as triethylamine interacts to a

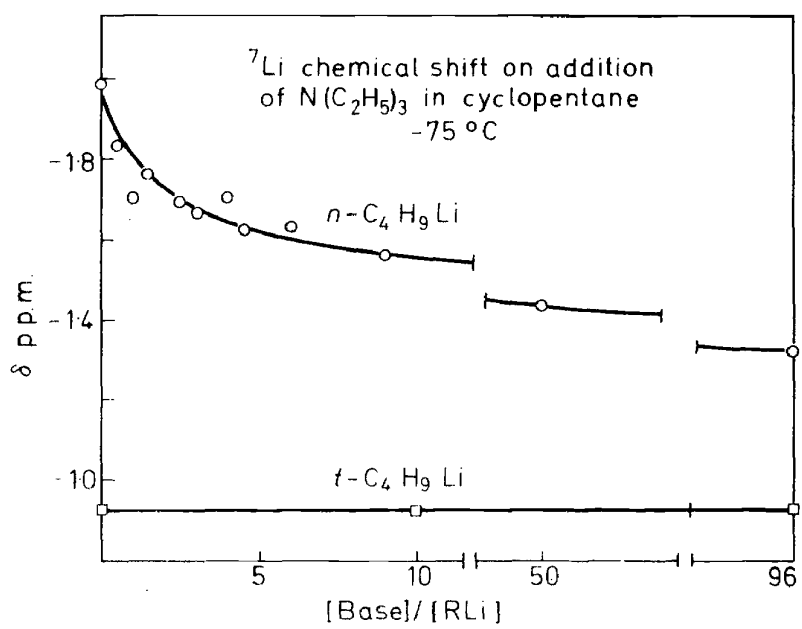

Figure 4. Effect of added triethylamine on the ${ }^{7} \mathrm{Li}$ chemical shift of $n$-butyllithium and $t$-butyllithium in cyclopentane at $-75^{\circ} \mathrm{C}$. In both cases the initial alkyllithium concentration was about $0 \cdot 1 \mathrm{M}$.

significant extent with the tert-butyllithium tetramer. Indeed, the bulkiness of the alkyl groups would seem to preclude much interaction of any base with the lithium sites in the intact tetramer. The marked acceleration in exchange on addition of base must therefore be related to interaction of the base with the transition state in the (presumably) dissociative process. This in turn suggests that the transition state probably resembles the dissociated species, i.e. dimers, in which there would be much more opportunity for lithiumbase interaction. The reaction profiles for dissociation of methyllithium in ether and tert-butyllithium in cyclopentane are shown in Figure 5, along with a suggested pathway for dissociation of the latter in the presence of base. It should be noted in this connection that methyllithium is undoubtedly quite strongly solvated as tetramer. If it were not for this solvation, methyllithium would not be soluble in basic solvents. It is reasonable to expect that a dimeric methyllithium moiety would be even more highly solvated but the fact that tetramer is the predominant species suggests that the additional solvation, even in THF solution, is not sufficient to overcome the strong bridge bonding found in the tetramer.

In contrast to tert-butyllithium, all other organolithium compounds studied so far in hydrocarbon solvents seem to undergo fairly rapid inter- 


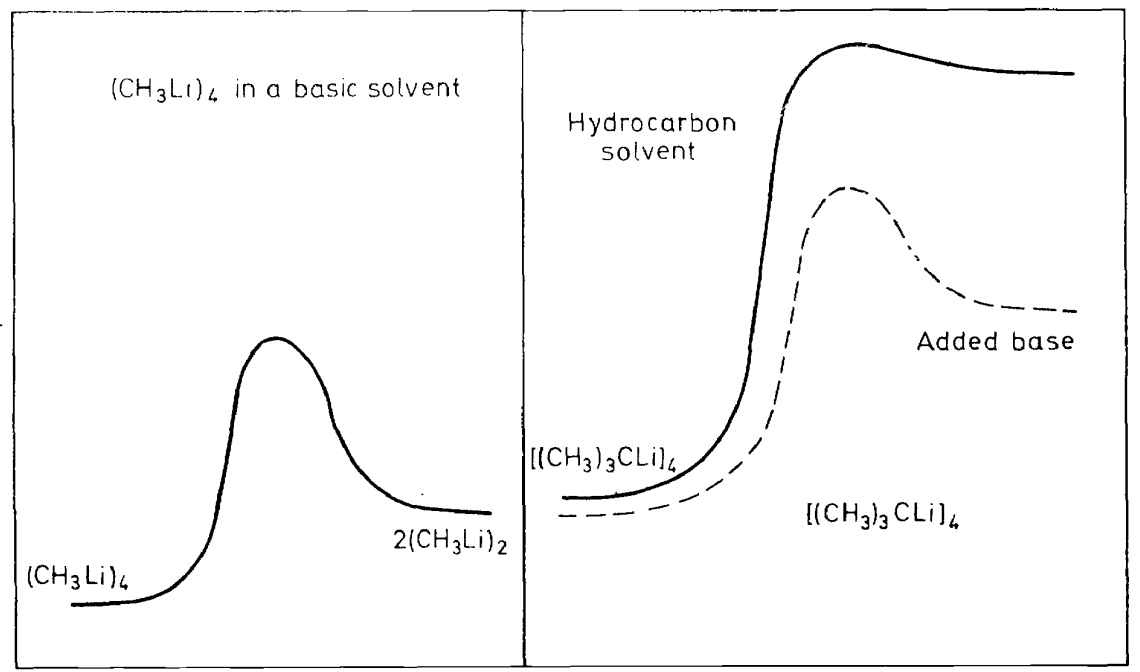

Figure 5. Free energy profiles for dissociation of methyllithium and tert-butyllithium.

molecular exchange in hydrocarbon solvents. The evidence for this statement consists in the observation of the temperature dependences of ${ }^{7} \mathrm{Li}$ multiplet spectra in hydrocarbon solvents ${ }^{12}$, and in the failure to observe a ${ }^{7} \mathrm{Li}-{ }^{13} \mathrm{C}$ scalar coupling ${ }^{16}$ for $n$-butyllithium in cyclohexane solution, whereas the coupling is observed for $t$-butyllithium in the same solvent. These other organolithium compounds either are hexameric in the solvent or there is some evidence which suggests that the hexamer does not differ greatly in free energy from the tetramer, even though the latter may predominate. For example ${ }^{6}$, trimethylsilylmethyllithium is tetrameric in benzene solution, with some tendency to shift toward hexameric in more concentrated solution, and is hexameric in cyclohexane solution. It would appear therefore, that the hexamer provides a low energy pathway for exchange. This may occur as a result of dissociation of the hexamer to form tetramer and dimer

$$
\mathrm{R}_{6} \mathrm{Li}_{6} \rightarrow \mathrm{R}_{4} \mathrm{Li}_{4}+\mathrm{R}_{2} \mathrm{Li}_{2}
$$

The dimer would then be picked up rapidly by a tetramer, or interact with a hexamer in a variety of possible ways:

$$
\begin{gathered}
\mathrm{R}_{2} \mathrm{Li}_{2}+\mathrm{R}_{4} \mathrm{Li}_{4} \rightarrow \mathrm{R}_{6} \mathrm{Li}_{6} \\
\mathrm{R}_{2} \mathrm{Li}_{2}+\mathrm{R}_{6} \mathrm{Li}_{6} \rightarrow 2 \mathrm{R}_{4} \mathrm{Li}_{4} \\
\mathrm{R}_{2} \mathrm{Li}_{2}+\underline{\mathrm{R}}_{6} \underline{\mathrm{Li}}_{6} \rightarrow \mathrm{R}_{6} \mathrm{Li}_{6}+\underline{\mathrm{R}}_{2} \underline{\mathrm{Li}}_{2}
\end{gathered}
$$

Since the hexamer might rupture to yield tetramer and dimer in any of three equivalent ways, the processes indicated are pathways for intermolecular exchange.

In summary, the observation of a rapid exchange in the absence of coordinating solvent or added base appears to be related to the presence of at least a small amount of hexamer which acts as a rapid transfer agent for 
dimeric alkyllithium fragments. Dissociation of tetrameric units is, by contrast, expected to be slower by many orders of magnitude, although it may still be rapid with respect to the observed rates of chemical reaction of alkyllithium compounds with substrates in hydrocarbon solvents.

Atoms other than carbon may act as bridging groups in organolithium systems. This fact is of relevance in understanding certain aspects of the chemical behaviour of alkyl- and aryl-lithium reagents. For example, when an alkyllithium solution is formed by reaction of an alkyl halide and lithium metal,

$$
\mathrm{RX}+2 \mathrm{Li} \rightarrow \mathrm{RLi}+\mathrm{LiX}
$$

the lithium halide formed in the reaction may form complexes with the organolithium moieties ${ }^{19}$. Figure 6 shows ${ }^{7}$ Li n.m.r. spectra of several mixtures of methyllithium and lithium bromide in diethyl ether ${ }^{20}$, at $-70^{\circ} \mathrm{C}$. In addition to the resonance at lowest field, due to methyllithium tetramer, several other absorptions occur, with relative intensities which depend on the ratio of

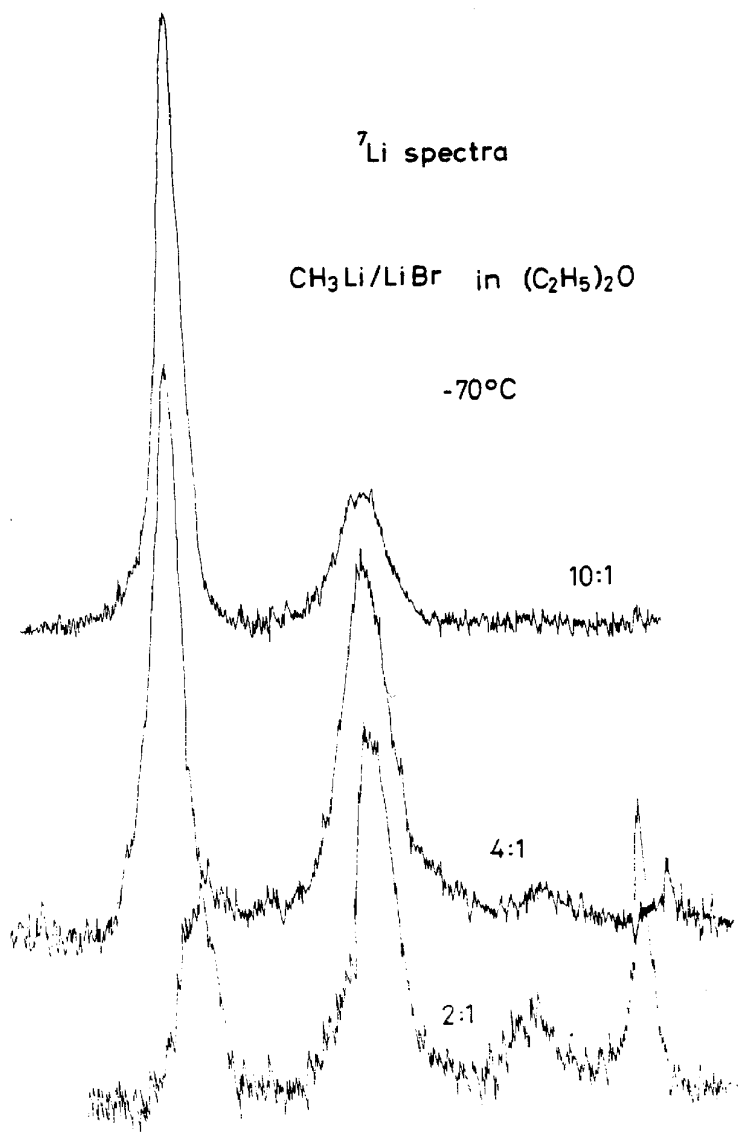

Figure 6. ${ }^{7} \mathrm{Li} \mathrm{n.m.r.} \mathrm{spectra} \mathrm{at} 38 \mathrm{MHz}$ of mixtures of methyllithium and lithium bromide ${ }^{20}$. 
$\mathrm{RLi}$ to LiX. Some of these have been fairly well identified (e.g. the absorption adjacent to the methyllithium peak is due to $\mathrm{R}_{3} \mathrm{XLi}_{4}$ ), but the compositions of all species present in the mixtures rich in $\mathrm{LiBr}$ are incompletely determined as yet. It can be said with considerable confidence, however, that methyllithium tetramers do not predominate in such solutions. Clearly, kinetic studies in which alkyllithium solutions rich in lithium bromide or iodide are employed are not likely to yield data which can be interpreted in a straightforward manner.

The presence of alkoxide bridges, arising from the reaction of the alkyllithium reagent with adventitious oxygen, is a commonly encountered difficulty which may have important kinetic consequences.

The compound lithium bis(trimethylsilyl)amide, $\mathrm{LiN}\left[\mathrm{Si}\left(\mathrm{CH}_{3}\right)_{3}\right]_{2}$, is an interesting example of a nitrogen bridging system ${ }^{21}$. The compound behaves very similarly to benzyllithium insofar as solubility, and degree of association (Table 1) are concerned. It is comparatively unreactive, even with THF. The compound provides a rather interesting example of the effects of solvation, in that it exists as a mixture of monomer and dimer in diethyl ether. The degree of association, as determined by isopiestic measurements ${ }^{8}$, is between one and two, depending on concentration. The ${ }^{7} \mathrm{Li}$ spectra, and ${ }^{1} \mathrm{H}$ spectra due to the methyl groups attached to silicon, exhibited the presence of two species. The concentration dependence of the relative intensities of these two species identifies which is due to monomer, which due to dimer, Figure 7. Measurements of the relative intensities of the absorptions as a function of temperature show that $\Delta H$ and $\Delta S$ for the equilibrium

$$
\left\{\mathrm{LiN}\left[\mathrm{Si}\left(\mathrm{CH}_{3}\right)_{3}\right]_{2}\right\}_{2} \rightleftarrows 2 \mathrm{LiN}\left[\mathrm{Si}\left(\mathrm{CH}_{3}\right)_{3}\right]_{2}
$$

are $-4.0 \mathrm{kcal} / \mathrm{mole}$ and $-20 \mathrm{cal} / \mathrm{deg}$-mole, respectively. These values can be interpreted as reflecting the role of the solvent in preferentially solvating the lithium atoms of the more dissociated species.

In hydrocarbon solutions it is also possible to discern two absorptions in the ${ }^{1} \mathrm{H}$ spectra at low temperatures. Colligative property data suggest, however, that the equilibrium in this case is between dimer and tetramer. In the absence of strong solvation of lithium, the enthalpy of dissociation of tetramers to dimers is positive. Finally it should be noted that the formation of strong bridge bonds, whether by carbon or another element, is associated with localization of charge at the bridging atoms. West and Waack ${ }^{7}$ have pointed this out in their comparison of the degrees of association of alkyllithium (tetramer), phenyllithium (monomer-dimer) and benzyllithium (monomer) in basic solvents. Similar considerations probably also apply to nitrogen and oxygen bridged systems, although information is lacking. Lithium $t$-butoxide is hexameric in solution and in the vapour phase ${ }^{11}$. Lithium(bis-trimethylsilyl)amide, on the other hand, appears to be more closely related to benzyllithium, which is much less extensively associated. Delocalization of charge in the amide ion might be responsible for this behaviour. Interaction of the $p_{n}$ orbital of nitrogen with the vacant $d_{\pi}$ orbitals of silicon might result in removal of charge from the bridging nitrogen.

Structural considerations provide the basis for models in terms of which kinetic observations might be rationalized. Organolithium compounds 


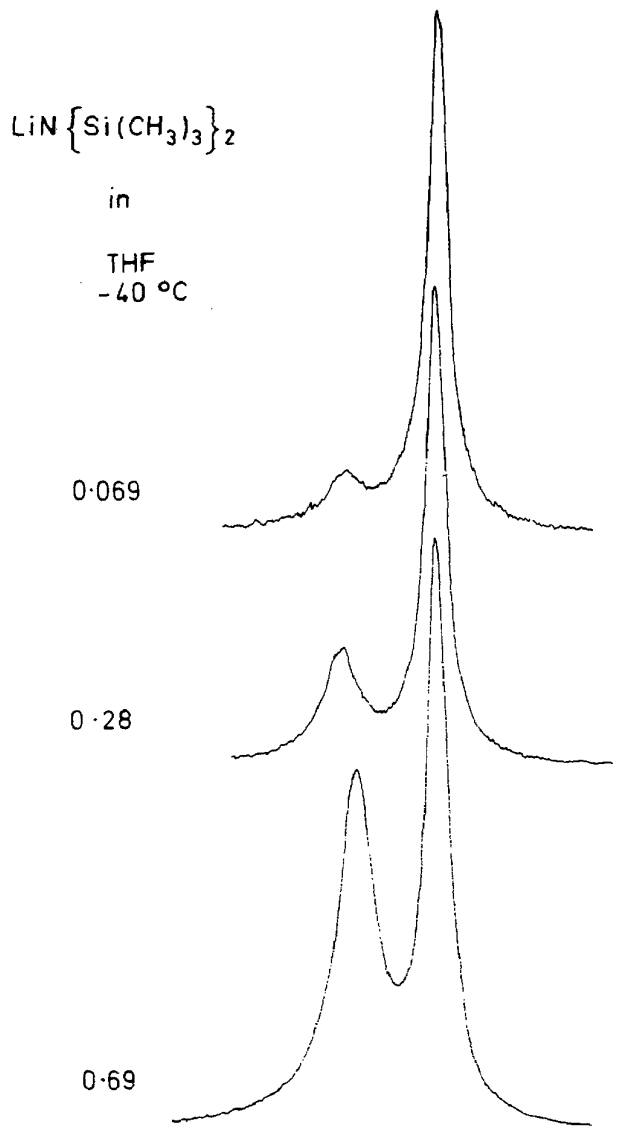

Figure 7. Concentration dependence of the ${ }^{7} \mathrm{Li}$ spectra of $\mathrm{LiN}\left[\left(\mathrm{Si}_{(\mathrm{CH}}\right)_{3}\right]_{2}$ in THF.

might be expected to react with substrates by one of a number of pathways. Among the more important possibilities are the following:

A. Reaction as carbanions-Reaction with a substrate might involve prior dissociation of a carbanion, followed by rapid attack of the carbanion on the substrate:

$$
\begin{aligned}
& \mathrm{R}_{n} \mathrm{Li}_{n} \rightarrow \mathrm{R}_{n-1} \mathrm{Li}_{n}^{+}+\mathrm{R}^{-} \\
& \mathrm{R}^{-}+\mathrm{S}_{u} \rightarrow \mathrm{Y}^{-} \\
& \mathrm{Y}^{-}+\mathrm{R}_{n-1} \mathrm{Li}_{n}^{+} \rightarrow \mathrm{R}_{n-1} \mathrm{YLi}_{n}
\end{aligned}
$$

Alternatively, there might be coordination of a nucleophilic substrate, as exemplified by a carbonyl group, with $\mathrm{R}_{n-1} \mathrm{Li}_{n}^{+}$:

$$
\begin{aligned}
& \mathrm{R}_{n-1} \mathrm{Li}_{n}^{+}+\mathrm{O}=\mathrm{C}^{\prime} \backslash \mathrm{R}_{n-1} \mathrm{Li}_{n}-\mathrm{O}-\stackrel{+}{\mathrm{C}}= \\
& \mathrm{R}_{n-1} \mathrm{Li}_{n}-\stackrel{+}{\mathrm{OC}} /+\mathrm{R}^{-} \rightarrow \mathrm{R}_{n-1} \mathrm{Li}_{n}-\mathrm{OC}_{\mathrm{R}}^{\prime}
\end{aligned}
$$


The fact that species of the form $\mathrm{R}_{n-1} \mathrm{Li}_{n}^{+}$are seen in high abundance in the mass spectrum, to the complete exclusion of the parent molecular ion $\mathrm{R}_{n} \mathrm{Li}_{n}^{+}$, might be taken as indication that an ionic dissociative process is not out of the question, especially in a fairly polar, solvating medium. The observation that inversion of configuration at the lithium-bearing carbon occurs in certain cases on the n.m.r. time scale $\left(10 \mathrm{sec}^{-1}\right)$ is evidence ${ }^{22}$ that carbanions, or incipient carbanions in the form of contact ion pairs, may form readily. The reactivity of a carbanion toward substrate of course depends on both the nature of the carbanion and the electrophilicity of the substrate. Where the processes described in the equations 11 or 12 are important pathways for reaction, and assuming that carbanions are formed in a rapid pre-equilibrium step, the reaction rate should exhibit a first order dependence on substrate, and a half-order dependence on organolithium. In addition, it should exhibit a marked acceleration due to strongly solvating solvent or added base.

B. Reaction as intact organolithium species - Direct reaction of the prevalent organolithium oligomer with substrate,

$$
\mathrm{B}_{m} \cdot \mathrm{R}_{n} \mathrm{Li}_{n}+\mathrm{S}_{u} \rightarrow \text { product }
$$

including a number $m$ of solvating base molecules, B, if these are present, would yield a first order kinetic dependence on organolithium and first order dependence on substrate. Since exchanges are invariably rapid in the presence of base, dissociated species (dimers, monomers) are present in the solution when bases are present. There must be a competition, therefore, between the dissociation products, which might be expected to have higher reactivity, and the parent species for reaction with substrate. In the absence of coordinating bases the steady state concentration of dissociated species should be much lower. On the other hand, their relative reactivity should be greater, since there is no base present to coordinate to the lithiums. Very few kinetic results have been reported which might be interpreted in terms of a reaction of the intact organolithium oligomer. Bartlett, Goebel and Weber ${ }^{23}$ have studied the ethylenation of secondary and tertiary alkyllithium compounds in the presence of various bases, and have concluded that the transition state for the reaction involves the intact, coordinated alkyllithium tetramer and one molecule of ethylene.

C. Reaction of dissociated organolithium species-Assuming dissociation of $\mathrm{R}_{n} \mathrm{Li}_{n}$ to monomers, and reaction of the monomer with substrate, the rate law for the reaction should be of the form ${ }^{24}$

$$
\text { Rate }=k K_{\mathrm{e}}^{1 / n}\left(\mathrm{R}_{n} \mathrm{Li}_{n}\right)^{1 / n}\left(\mathrm{~S}_{u}\right)
$$

Rate laws which are fractional order in alkyllithium and first order in substrate have been observed in a number of systems. Waack and Doran ${ }^{25}$, for example, have observed the reaction of a number of organolithium compounds with 1,1-diphenylethylene in THF, and have found effective reaction orders of 1 for allyllithium and benzyllithium, 0.27 for methyllithium, 0.66 for phenyllithium and 0.34 for vinyllithium. The results for the benzyllithium and methyllithium are consistent with the presumed degrees of association in THF based on other observations (Table 1). The association of vinyllithium is not 
known. Phenyllithium is thought to be dimeric. The observed order for the latter compound is possibly due to competitive reactions of both monomer and dimer.

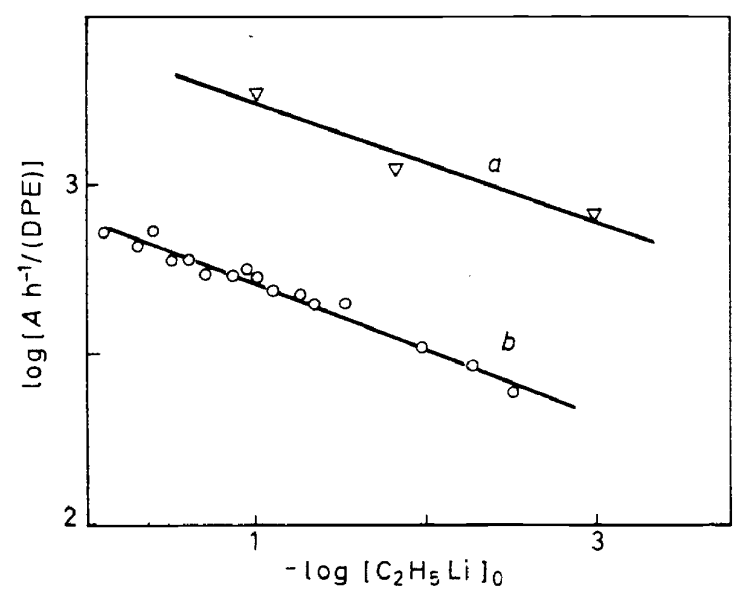

Figure 8. $\log$ (initial rate) versus $\log$ initial [R Li] for reaction of (a) $n$-butyllithium in benzene at $30 \cdot 5^{\circ} \mathrm{C}^{26}$, and (b) ethyllithium in benzene at $25 \cdot 0^{\circ} \mathrm{C}^{27}$, with 1,1 -diphenylethylene.

An early study by Evans and George ${ }^{26}$ of the reaction of $n$-butyllithium with 1,1-diphenylethylene in benzene also suggested a low reaction order in alkyllithium, as determined by a graph of $\log$ (initial rate) versus $\log [\mathrm{RLi}]_{0}$, Figure 8. More complete studies in our laboratories of the reaction of ethyllithium with 1,1-diphenylethylene in benzene ${ }^{27}$ confirm the low reaction order over a wide range of alkyllithium concentration. It would appear that in these systems the monomeric alkyllithium species predominates in the kinetics. It is important to note, however, that in the studies carried out in hydrocarbon solvent, and in the initiation of styrene polymerization ${ }^{24}$, the organolithium compound is usually present in considerable excess. When this is not so, the assumption of a rapid pre-equilibrium may not be valid $^{5}$, and the reaction may exhibit complex behaviour.

D. Radical reaction pathways-It has been known for some time that in a number of special systems, radicals are involved in the reactions of organolithium compounds ${ }^{28,29}$. In more recent years, the work of D'yachkovskii and Shilov ${ }^{30}$ and co-workers has demonstrated that radicals are involved in the reactions between ethyllithium and a number of alkyl halides. The recent discovery of the phenomenon of chemically induced dynamic nuclear polarization (CIDNP) ${ }^{31,32}$ in reactions of organolithium compounds with alkyl halides has provided strong additional evidence for radical character in the coupling reaction and in the halogen-metal interchange ${ }^{33-35}$. At this early stage it is not yet possible to assess the full potential of CIDNP for elucidating the detailed nature of organometallic reactions. Even the few results presently available, however, are of significance for an understanding of the pathways by which the coupling and interchange reactions occur. 
For more details regarding the basis of the experiment the reader is referred to the references listed ${ }^{32,36,37}$.

The CIDNP phenomenon is perceived by observing the p.m.r. spectrum of a chemically reacting system involving, for example, an alkyllithium compound and an alkyl halide. Among the products of such a reaction mixture are the coupling product, and disproportionation products of the two radicals which might be formed, e.g.

$$
n-\mathrm{C}_{4} \mathrm{H}_{9} \mathrm{Li}+n-\mathrm{C}_{4} \mathrm{H}_{9} \mathrm{Br} \rightarrow \mathrm{C}_{4} \mathrm{H}_{10}, \mathrm{C}_{4} \mathrm{H}_{8} \text {, etc. }
$$

The p.m.r. spectra of reacting mixtures show, under the appropriate conditions, strongly enhanced absorptions or strong emissions, in the lines due to the coupling products, particularly in the olefin formed in the disproportionation reaction. In addition, the lines due to the alkyl halides show the same sort of effects, and when a metal-halogen interchange can be detected by using RLi and $\mathrm{R}^{\prime} \mathrm{I}$, both the original alkyl halide and the new one produced by metal-halogen interchange show, concurrently, the enhanced absorption or emission ${ }^{33,35}$.
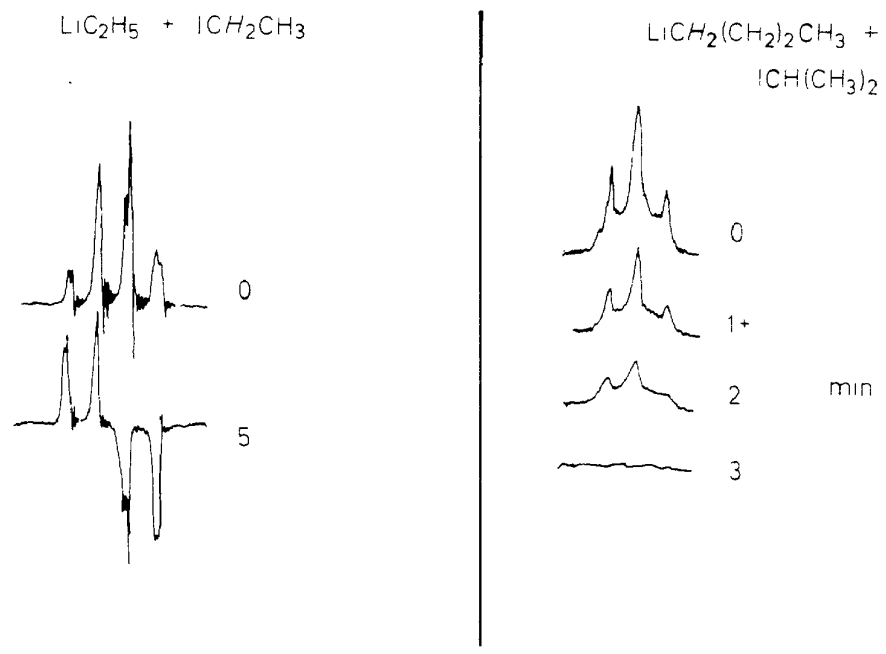

Figure 9. Proton magnetic resonance spectra of (a) the $\mathrm{CH}_{2}$ quartet of ethyl iodide in ethyllithium and 2-iodobutane in hexane and (b) $-\mathrm{CH}_{2}$ quartet of $n$-butyllithium in $n$-butyllithium + 2 -iodopropane in hexane ${ }^{37}$.

It had been pointed out some time ago by Applequist and $\mathrm{O}^{\prime} \mathrm{Brien}^{38}$ that in the typical halogen-metal interchange reaction the concurrent formation of radicals characteristic of both the halide and lithium reagent was required to account for certain of the results. The CIDNP results recently reported by Ward, Lawler and Cooper ${ }^{33}$ and by Lepley ${ }^{34,35}$ confirm that this is so. Thus, in Figure 9 are shown the $\mathrm{CH}_{2}$ quartet lines of ethyl iodide a few minutes after mixing ethyllithium and 2 -iodobutane in hexane ${ }^{35}$. The same sort of phenomenon is observed in the $\mathrm{CH}$ sextet of the reacting 2iodobutane. Thus it is necessary to formulate a model for the reaction which 
makes it possible to form an intermediate radical from either alkyl group, so that whichever forms alkyl halide in the ensuing step will exhibit dynamic nuclear polarization.

A second aspect of the systems which have been studied in hydrocarbon solutions relates to the observation that the absorptions due to the organolithium reagent does not exhibit nuclear polarization. We have examined a number of alkyllithium-alkyl iodide systems in hydrocarbon solutions, and find that neither the proton nor ${ }^{7} \mathrm{Li}$ resonances of the alkyllithium species shows polarization effects.

It has been noted that alkyl radicals produced in reactions with alkyl halides are considerably less reactive than free alkyl radicals, presumably because of complex formation of some sort with the oligomeric alkyllithium species $^{30,39}$. This observation, together with the known exchange properties of the alkyllithium systems, provides a basis on which to propose a model for the reaction between an alkyllithium compound and alkyl halide which is consistent with the nuclear polarization observations.

Although kinetic data are extremely limited, the present indications are that the reaction between an alkyllithium compound and an alkyl halide in hydrocarbon solvent to give the coupling product is first order in each reactant $\mathrm{t}^{40,41}$. We assume, therefore, that there is an initial interaction between the oligomeric organolithium compound and the alkyl halide, to form a weak complex

$$
\mathrm{R}_{n} \mathrm{Li}_{n}+\mathrm{R}^{\prime} \mathrm{X} \rightleftarrows \mathrm{R}_{n} \mathrm{Li}_{n} \cdot \mathrm{XR}^{\prime}
$$

This complex may be thought of as a weak charge transfer type, so that the ground state for it contains some element of a form $\mathrm{R}_{n} \mathrm{Li}_{n}^{+} \cdot \mathrm{X}^{-}-\mathrm{R}^{\prime}$. We propose that the step which results in formation of alkyl radicals involves electron transfer from $\mathrm{R}_{n} \mathrm{Li}_{n}$ to $\mathrm{XR}^{\prime}$, followed by shifting of $\mathrm{X}^{-}$to a face of the alkyllithium oligomer, with simultaneous formation of two radicals, $R$ and $\mathbf{R}^{\prime}$, as depicted in Figure 10. Transfer of an electron to $\mathrm{X}$ from one of the adjacent four centre bonds should result in ready cleavage of the $X-R^{\prime}$ bond. It is very reasonable that $R$ should be bost as a radical also, inasmuch as the incipient $\mathrm{X}^{-}$readily occupies a bridging position, as noted earlier. Furthermore, the tendency of the $\mathrm{R}_{n} \mathrm{Li}_{n}^{+}$to lose a radical to form $\mathrm{R}_{n-1} \mathrm{Li}_{n}^{+}$is manifested in the mass spectral results. We have then provided for concurrent formation of $\mathrm{R}_{n-1} \mathrm{Li}_{n} \mathrm{X}+\mathrm{R}+\mathrm{R}^{\prime}$. Coupling of $\mathrm{R}$ and $\mathrm{R}^{\prime}$, or other radical disproportionation reactions should follow rapidly, with the resultant appearance of nuclear polarization in the spectra of the products. The process leading to formation of the two alkyl radicals, may, however, be reversed, at least in part. Attack of either R or $\mathbf{R}^{\prime}$ on X to form RX or $\mathbf{R}^{\prime} \mathbf{X}$, respectively, would result in a partial restoration of the original conditions, with the important difference, however, that the alkyl halide thus formed is located on one of the faces of the newly formed $R_{n-1} L_{n}$ radical. If this newly formed alkyl halide remains on the face of the alkyllithium radical cluster, it prevents recombination of $\mathbf{R}_{n-1} \mathrm{Li}_{n}$ with $\mathbf{R}$ or $\mathbf{R}^{\prime}$, as the case may be.

From the exchange work which has been carried out to date the characteristic time for exchange at room temperature involving hexameric species, e.g. the transfer of a dimer from a hexamer to a tetramer, is not well known. 

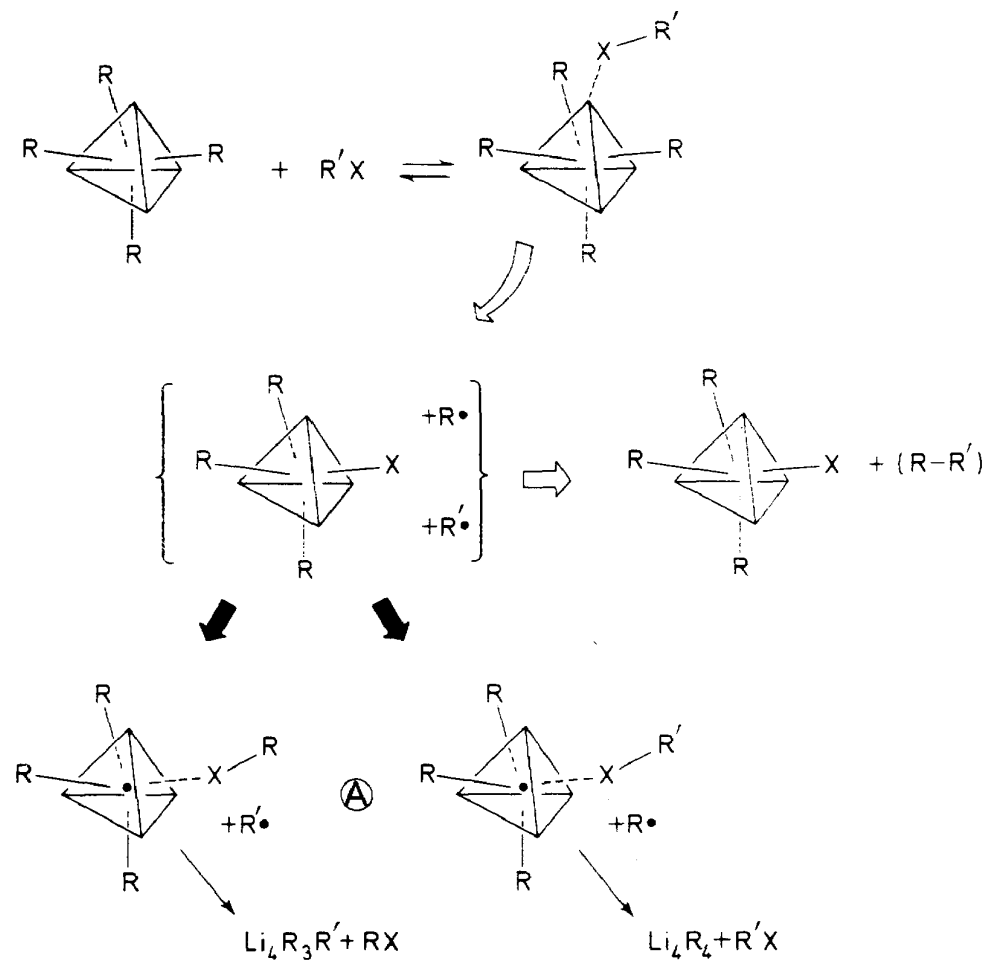

Figure 10. A possible pathway for coupling and halogen-metal interchange in the reaction of alkyllithium with alkyl halide ${ }^{42}$.

It is possible to estimate, however, that the inverse rate constant for such a process is on the order of perhaps $10^{-3}$ to $10^{-2} \mathrm{sec}$ for ethyllithium, for example, in a hydrocarbon solvent or in benzene. If the complex labelled as A in Figure 10, involving the two radicals which are prevented from recombination by the complex formation, persists for periods of up to $10^{-2}$ sec, exchanges may occur during the lifetime of the species. The effect of these exchanges will be to expose a larger number of organolithium protons to the radical spin. It may be possible, in an appropriate experiment, to verify the existence of alkyllithium-radical complexation through its effect on the nuclear spin relaxations.

\section{ACKNOWLEDGEMENT}

This research was supported by a grant, GP 6396X, from the National Science Foundation. The ${ }^{7} \mathrm{Li}$. m.r. spectra were obtained from an instrument purchased under a research instrument grant from the National Science Foundation. 


\section{THEODORE L. BROWN}

\section{REFERENCES}

${ }^{1}$ H. Dietrich, Acta. cryst., Camb. 16, 681 (1963).

2 E. Weiss and E. A. C. Lucken, J. Organometal. Chem. 2, 197 (1964).

${ }^{3}$ S. P. Patterman, I. L. Karle and G. D. Stucky, J. Am. Chem. Soc. 92, 1150 (1970).

4 G. D. Stucky. Private communication.

5 T. L. Brown, Advanc. Organometal. Chem. 3, 365-395 (1966).

${ }^{6}$ H. L. Lewis and T. L. Brown, J. Am. Chem. Soc. 92, 4664 (1970).

7 P. West and R. Waack, J. Am. Chem. Soc. 89, 4395 (1967).

8 B. Y. Kimura and T. L. Brown, J. Organometal. Chem. In press.

9 M. Weiner, C. Vogel and R. West, Inorg. Chem. 1, 654 (1962).

10 J. Berkowitz, D. A. Bafus and T. L. Brown, J. Phys. Chem. 65, 1380 (1961).

11 G. E. Hartwell and T. L. Brown, Inorg. Chem. 5, 1257 (1966).

${ }^{12}$ M. Y. Darensbourg, B. Y. Kimura, G. E. Hartwell and T. L. Brown, J. Am. Chem. Soc. 92 , $1237(1970)$.

${ }^{13}$ L. M. Seitz and T. L. Brown, J. Am. Chem. Soc. 88, 2174 (1966).

14 T. L. Brown, Accts. Chem. Res. 1, 23 (1968).

15 L. D. McKeever, R. Waack, M. A. Doran and E. B. Baker, J. Am. Chem. Soc. 91, 1057 (1969).

16 L. D. McKeever and R. Waack. Private communication.

17 T. L. Brown, B. Y. Kimura and L. M. Seitz, J. Am. Chem. Soc. 90, 3245 (1968).

18 K. C. Williams and T. L. Brown, J. Am. Chem. Soc. 88, 4134 (1966).

19 R. Waack, M. A. Doran and E. B. Baker, Chem. Commun. 1291 and 1968 (1967).

20 D. P. Novak and T. L. Brown. Unpublished observations.

21 U. Wannagat and H. Niederprum, Chem. Ber. 94, 1540 (1961).

22 M. Witanowski and J. D. Roberts, J. Am. Chem. Soc. 88, 736 (1966).

23 P. D. Bartlett, C. V. Goesel and W. P. Weber, J. Am. Chem. Soc. 91,7425 (1969).

24 S. Bywater and D. J. Worsfold, Canad. J.Chem.. 38, 1891 (1960).

25 R. Waack and M. A. Doran, J. Am. Chem. Soc. 91, 2456 (1969).

26 A. G. Evans and D. B. George, J. Chem. Soc. $4653-4659$ (1961).

27 M. Y. Darensbourg and T. L. Brown. Unpublished results.

28 D. Bryce-Smith, J. Chem. Soc. 1603 (1956).

29 R. Waack and M. A. Doran, J. Organometal. Chem. 3, 94 (1965).

${ }^{30}$ F. S. D'yachkovskii and A. E. Shilov, Russ. Chem. Revs. 35, 300 (1966).

${ }^{31}$ H. Fischer and J. Bargon, Accts. Chem. Res. 2, 110 (1969).

32 H. R. Ward and R. G. Lawler, J. Am. Chem. Soc. 89, 5518 (1967).

${ }^{33}$ H. R. Ward, R. G. Lawler and R. A. Coper, J. Am. Chem. Soc. 89, 5518 (1967).

34 A. R. Lepley and R. L. Landau, J. Am. Chem. Soc. 91, 748 (1969).

35 A. R. Lepley, Chem. Commun. 64 (1969).

${ }^{36}$ R. Kaptein, Chem. Phys. Ltrs, 2, 261 (1968).

${ }^{37}$ G. L. Closs, J. Am. Chem. Soc. 91, 4552 (1969).

38 D. E. Applequist and D. F. O'Brien, J. Am. Chem. Soc. 85, 743 (1963).

39 Radicals have also been observed directly in e.s.r. experiments; H. Fischer, J. Phys. Chem. 73, 3834 (1969); G. A. Russell and D. W. Lamson, J. Am. Chem. Soc. 91, 3967 (1969).

40 R. West and W. Glaze, J. Chem. Phys. 34, 685 (1961).

41 F. A. Settle Jr. Ph.D. Thesis, University of Tennessee (1964) reports the kinetics of halogenmetal interchange between butyllithium and ethyl iodide in ether.

42 The mechanism here is in some respects similar to the SET (single electron transfer) mechanism suggested by Screttas and Eastham ${ }^{43}$, although there is not a solvent support of such transfer as in the systems discussed by them.

43 C. G. Screttas and J. F. Eastham, J. Am. Chem. Soc. 88, 5668 (1966). 